\title{
A New Design for Noise-Induced Chattering Reduction in Sliding Mode Control
}

\author{
Min-Shin Chen ${ }^{1}$ and Ming-Lei Tseng ${ }^{2}$ \\ ${ }^{1}$ National Taiwan University \\ ${ }^{2}$ Chinese Culture University \\ Taiwan, Republic of China
}

\section{Introduction}

The sliding mode control (Utkin, 1977; Hung et al., 1993) is robust with respect to certain structured system uncertainties and disturbances. However, the early version of sliding mode control adopts a switching function in its design, and this results in high-frequency oscillations (the so-called chattering) in the control signal. Such control chattering is undesirable since it can damage the actuator and the system. Among the various solutions to reducing chattering, the boundary layer design (Burton \& Zinober, 1986; Slotine \& Sastry, 1983) is probably the most common approach. In the boundary layer design, a smooth continuous function is used to approximate the discontinuous sign function in a region called the boundary layer around the sliding surface. As a result, the control signal in a boundary layer design will contain no chattering in a noise free environment. However, the boundary layer design has two disadvantages. First, chattering reduction of the control signal is achieved at the sacrifice of control accuracy. To obtain smoother control signals, one must adopt a larger boundary layer width. But a larger boundary layer width results in larger errors in control accuracy. Second, when there is high-level measurement noise, the boundary layer design becomes ineffective in chattering reduction.

One of the purposes of this chapter is to show that contrary to the common belief, the boundary layer design does not completely solve the chattering problem in practical applications. Essentially this is due to the fact that the boundary layer control design is still a high gain design, and as a result, its control signal is very sensitive to high-frequency measurement noise. Control chattering may still take place due to the excitations of measurement noise. This fact will be demonstrated via the frequency domain analysis.

The other purpose of this chapter is to present a new design for chattering reduction by low-pass filtering the control signal. The new design will be shown to be able to avoid the disadvantages of conventional boundary layer design while effectively reduce chattering. The new design adopts a special control structure, in which an integrator is placed in front of the system to be controlled. A sliding mode control $w$ is then constructed for the extended system (the original system plus the integrator). The control signal $w$ hence has chattering, but the true control signal $u$ going into the system is smooth since the high frequency chattering in $w$ will be filtered out by the integrator, which acts as a low pass filter. With such a design, the chattering reduction is achieved by low pass filtering, and at the same time the control accuracy can be maintained. Another advantage of the new design is that 
in noisy environment, measurement noise causes severe chattering in the signal $w$, but the integrator can still effectively filter out the chattering. Hence, the new design has better noise immunity than the conventional boundary layer design. Previous literature (Sira-Ramirez, 1993; Sira-Ramirez et al., 1996) contains no stability analysis or performance analysis, and does not address noise-induced chattering. In (Xu et al., 2004), two first-order filters are employed but again noise-induced chattering is not addressed.

The new low-pass-filtering design for chattering reduction is nevertheless non-trivial. As is known, the sliding variable in sliding mode control design must be chosen such that control input shows up in the time derivative of sliding variable. In this way, the control input can influence how the sliding variable evolves. Such a design guideline must also be observed in the new design. Hence, the time derivative of the new sliding variable for the extended system should contain the sliding mode control $w$. This in turn suggests that the new sliding variable itself for the extended system contains the integration of $w$ which is the true control signal $u$. Since the unknown disturbance $d$ enters the system in the same place as the control signal $u$ (the so-called matching condition (Corless \& Leitmann, 1981)), the new sliding variable will inevitably contains the unknown disturbance $d$, and this makes evaluation of the sliding variable difficult. This is a problem that is unique to the low-pass-filtering design. Previous literature (Bartolini, 1989; Bartolini \& Pydynowski, 1996) has attempted to solve this problem only with partial success. In (Bartolini, 1989), a variable structure estimator is proposed to estimate the sliding variable, but it must assume a priori that the system state is uniformly bounded before proving the system stability. In (Bartolini \& Pydynowski, 1996), a one-dimensional observer is proposed to estimate the sliding variable, but stability is guaranteed only if a differential inequality with bounded coefficients is satisfied. This chapter will propose a complete solution by using the disturbance estimator proposed in (Chen \& Tomizuka, 1989) for sliding variable estimation. A rigorous stability proof of the new sliding mode control will also be presented.

This chapter is organized as follows. Section 2 reviews the boundary layer design for the sliding mode control of a linear uncertain system. A simulation example is given to reveal the weakness of boundary layer design. Section 3 introduces the new chattering reduction control design. A second simulation example is given to confirm the advantage of new design. Finally, Section 4 gives the conclusions.

\section{Boundary layer control}

The purpose of this section is to review the boundary layer design in sliding mode control for a linear system with matching disturbance.

\subsection{Noise-free boundary layer control}

Consider a linear system with matching disturbance :

$$
\dot{x}_{0}=A x_{0}+B\left(u_{0}+d\right),
$$

where $x_{0} \in R^{n}$ is the system state available from noise-free measurement, $u_{0} \in R^{1}$ is the scalar control input, and $d$ is an unknown disturbance with known upper bounds $|d| \leqslant D_{0}$, $|\dot{d}| \leqslant D_{1}$. The system matrices $A \in R^{n \times n}$ and $B \in R^{n \times 1}$. The pair $(A, B)$ is controllable. The control objective is to eliminate the interference of the disturbance $d$ with the control $u_{0}$. 
To achieve this objective, a sliding mode control with boundary layer design has previously been proposed as :

$$
u_{0}=-K x_{0}+v_{0}
$$

where $K$ is the state feedback gain that places the poles of $(A-B K)$ to the left half plane so that there exists a positive definite matrix $P$ satisfying the Lyapunov equation

$$
(A-B K)^{T} P+P(A-B K)=-I,
$$

and $v_{0}$ is the boundary layer control :

$$
v_{0}=-\rho \frac{s_{0}}{\left|s_{0}\right|+\epsilon} \quad, \quad \rho>D_{0}
$$

with the sliding variable $s_{0}$ given by

$$
s_{0}=2 B^{T} P x_{0}
$$

and $\epsilon$ a small positive number specifying the boundary layer width. Since the above boundary layer control is a continuous function of the system state, the resultant control signal (2) will have no chattering phenomenon if there is no measurement noise and unmodeled dynamics. Note that close to the sliding surface $\left(s_{0} \approx 0\right)$, the boundary layer control $(4)$ reduces to a proportional control with high control gain: $v_{0}=-(\rho / \epsilon) s_{0}$. This high gain characteristics is the cause of noise-induced chattering introduced in the next section.

\subsection{Noise-corrupted boundary layer control}

In order to analyze how the conventional boundary layer control responds to measurement noise, a zero-mean stochastic noise $n$ is introduced into the measurement of system state $x$. The state equation (1) thus becomes :

$$
\dot{x}_{1}=A x_{1}+B\left(u_{1}+d\right),
$$

where $x_{1}$ is the noise-affected system state, $u_{1}$ is the noise-affected control input:

$$
u_{1}=-K\left(x_{1}+n\right)+v_{1}, \quad v_{1}=-\rho \frac{s_{1}}{\left|s_{1}\right|+\epsilon},
$$

where $n$ is the stochastic measurement noise, and $s_{1}$ the sliding variable

$$
s_{1}=2 B^{T} P\left(x_{1}+n\right) .
$$

Define the perturbed control input $\delta u=u_{1}-u_{0}$ as the difference between the noise-free $u_{0}$ in the previous section and noise-affected $u_{1}$ in this section. Similarly, the perturbed state $\delta x=x_{1}-x_{0}$ is the difference between the noise-free $x_{0}$ and noise-affected $x_{1}$. Since the measurement noise $n$ is assumed to be of small magnitude, so are $\delta u$ and $\delta x$. As a result, one can apply linearization technique to the nonlinear boundary layer control system; in particular, one can derive the linear transfer function $T_{n}(s)$ from the measurement noise $n$ to the perturbed control $\delta u$. From this transfer function $T_{n}(s)$, one can learn how the high-frequency measurement noise $n$ affects the perturbed control $\delta u$ and the noise-affected input $u_{1}=u_{0}+\delta u$. If the high-frequency gain of $T_{n}(s)$ is large, it suggests that measurement 
noise $n$ can induce high-frequency chattering in the control $u_{1}$. The derivation procedure of $T_{1}(s)$ is as follows.

It follows from (1) and (6)

$$
\delta \dot{x}=A \delta x+B \delta u
$$

and from (2) and (7),

$$
\delta u=-K(\delta x+n)+v_{1}-v_{0} .
$$

If one defines $f(x)=\rho \frac{2 B^{T} P x}{\left|2 B^{T} P x\right|+\epsilon}$, according to (4) and (7),

$$
v_{1}-v_{0}=-f\left(x_{1}+n\right)+f\left(x_{0}\right) \fallingdotseq-\frac{\partial f}{\partial x} x_{x=x_{0}} \cdot\left(x_{1}+n-x_{0}\right)=-N(\delta x+n),
$$

where the second equality results from the Taylor series expansion of $f\left(x_{1}+n\right)$ at $x_{0}$, and

$$
N=\frac{\partial f}{\partial x}_{x=x_{0}}=\rho \frac{2 B^{T} P \epsilon}{\left(\left|s_{0}\right|+\epsilon\right)^{2}} \in R^{1 \times n}
$$

Note that in the above Taylor series expansion of the nonlinear function $f(\cdot)$, one can neglect all high-order terms and retains only the first order term because $\delta x+n$ is small.

Combining equations (10) and (11) gives

$$
\delta u=-(K+N)(\delta x+n) .
$$

Substituting the above equation into (9) results in the closed-loop transfer function from $n$ to $\delta x$ :

$$
\delta x=-[s I-A+B(K+N)]^{-1} B(K+N) n,
$$

where $s$ represents the Laplace transform operator. Finally, the transfer function from $n \in R^{n}$ to $\delta u \in R^{1}$ can be deduced from (13) and (14),

$$
\delta u=T_{n}(s) n, \quad T_{n}(s)=\left\{(K+N)[s I-A+B(K+N)]^{-1} B-I\right\}(K+N) .
$$

One may now use Equation (15) to study how the stochastic measurement noise $n$ affects the control input $u_{1}=u_{0}+\delta u$ in the boundary layer control. In particular, one is interested in knowing whether the high-frequency measurement noise $n$ will contribute to the chattering (high-frequency oscillations) of control signals in a boundary layer design. Note that control chattering occurs only after the sliding variable $s_{0}$ approaches almost zero. When this occurs, the vector $N$ in (12) may be approximated by

$$
N \fallingdotseq \rho \frac{2 B^{T} P}{\epsilon} .
$$

One may now plot the Bode diagram of $T_{n}(s)$ in (15) with the row vector $N$ given as above to check how sensitive the boundary layer control is to the measurement noise.

A simulation example is given below to show that even if a boundary layer design has been used, control chattering may still take place due to the measurement noise. 
Example 1: Consider the system (1) with

$$
A=\left[\begin{array}{ccc}
0 & 1 & 0 \\
0 & 0 & 1 \\
7 & -1 & 2
\end{array}\right], \quad B=\left[\begin{array}{l}
0 \\
0 \\
1
\end{array}\right],
$$

and a disturbance $d=\cos (t)$. The sliding mode control (2) and (4) has design parameters: boundary layer width $\epsilon=1,0.01$, and 0.001 respectively, control gain $\rho=1.2$, and state feedback gain $K=\left[\begin{array}{lll}67 & 46 & 14\end{array}\right]$. From (15), the singular value of transfer function $T_{n}(s)$ from $n$ to $\delta u \in R^{1}$ is plotted in Figure 1 .

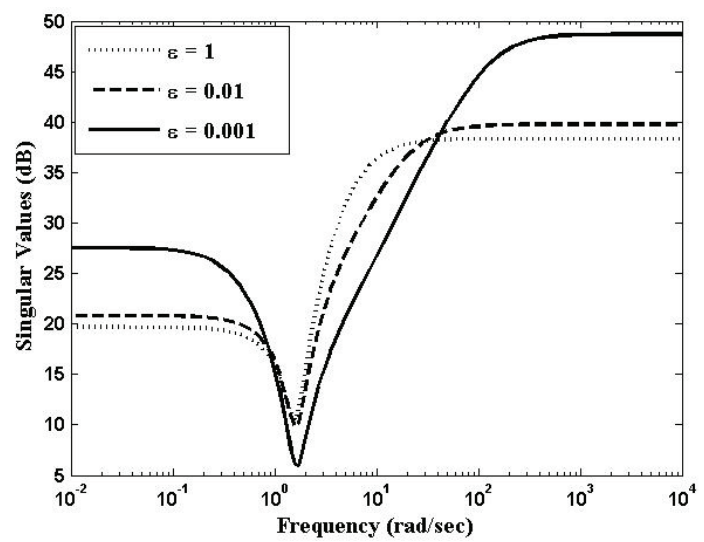

Fig. 1. Singular values of $T_{n}(s)$ with different $\epsilon$

The high gain of $T_{n}(s)$ at high frequency suggests that the sliding mode control signal is very sensitive to high-frequency measurement noise. The smaller the boundary layer width $\epsilon$, the more sensitive the control input to the measurement noise. As a result, the high frequency measurement noise $n$ will create substantial high frequency oscillations (chattering) in the perturbed control $\delta u$, and hence in the noise-affected input $u_{1}=u_{0}+\delta u$. Figure 2 shows the time response of control input $u_{1}$, which confirms the existence of high frequency chattering even if a boundary layer of width $\epsilon=0.005$ has been introduced into the sliding mode control design.

\section{Filtered sliding mode control}

\subsection{Sliding variable design}

As is demonstrated in the simulation example 1, sliding mode control with the boundary layer design still exhibits the chattering phenomenon when there is a high level of measurement noise. Hence, a solution better than the boundary layer design is required to reduce the chattering in sliding mode control. To this end, one will introduce the Filtered Sliding Mode Control in this section, whose control structure is depicted in Figure 3. In Figure 3, an integrator is intentionally placed in front of the system, and $w=\dot{u}$ is treated as the control variable for the extended system. A switching sliding mode control law is chosen for $w$ to suppress the effects of disturbance $d$. Even though $w$ is chattering, the control input $u$ to the system will be smooth because the high-frequency chattering will be filtered out by the 


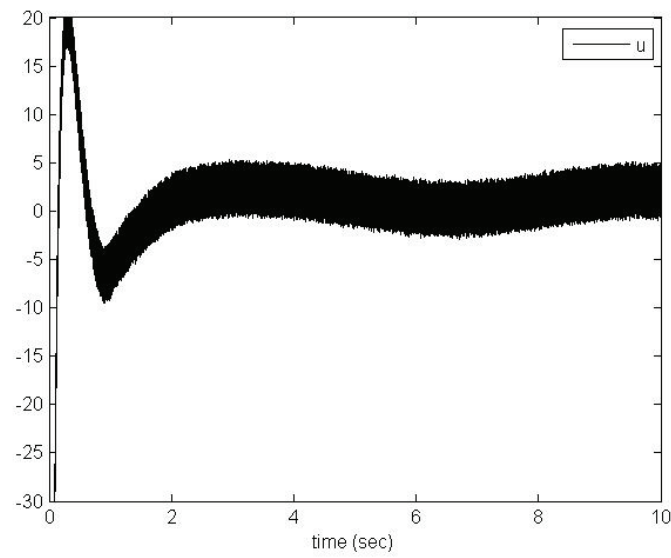

Fig. 2. Time history of control input

integrator, which acts as a low-pass filter. In other words, the new control design removes chattering by filtering the control signal, hence, the control structure in Figure 3 is called Filtered Sliding Mode Control.

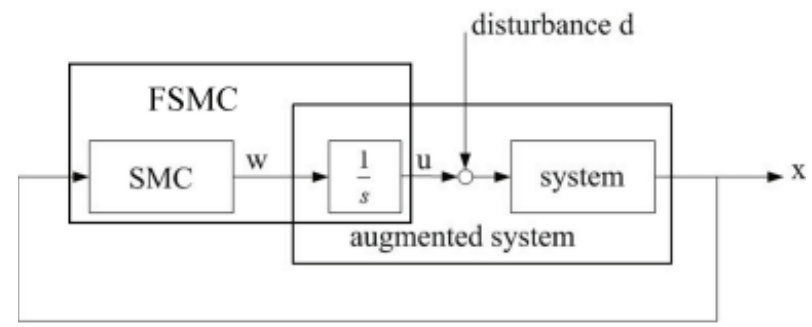

Fig. 3. Filtered sliding mode control

Consider a linear system with disturbance:

$$
\dot{x}=A x+B(u+d) .
$$

For the design of filtered sliding mode control, one chooses the sliding variable as follows.

$$
s_{2}=\dot{z}+\lambda z, \quad z=C x,
$$

where $\lambda$ is a positive constant, and the row vector $C \in R^{1 \times n}$ is chosen such that $(A, B, C)$ is of relative degree one, and the $(n-1)$ zeros of the system $(A, B, C)$ are in the stable locations. It will be shown in the proof of Theorem 3 below that when $s_{2}$ is driven to zero, the system state $x$ will also be convergent to zero.

Using (17) and (16), one finds

$$
s_{2}=C A x+C B(u+d)+\lambda C x,
$$


and, by taking the time derivative of $s_{2}$,

$$
\begin{aligned}
\dot{s}_{2} & =\left(C A^{2}+\lambda C A\right) x+(C A B+\lambda C B) u+C B w \\
& +(C A B+\lambda C B) d+C B \dot{d} .
\end{aligned}
$$

Note that the control variable $w=\dot{u}$ appears in the time derivative of the sliding variable $s_{2}$, suggesting that one can control the evolution of $s_{2}$ by properly choosing the control variable $w$. However, there is a problem that according to (18), the expression of $s_{2}$ contains the unknown disturbance term $d$. Therefore, it is difficult to evaluate the sliding variable $s_{2}$.

To solve this problem, one will use the Disturbance Estimator proposed in (Chen \& Tomizuka, 1989 ) to estimate the disturbance $d$. With an estimate of $d$, one can obtain an estimate of the sliding variable $s_{2}$ via (18). In the sequel, an estimator for the unknown disturbance $d$ will be constructed based on the scalar variable $z$ defined in (17). Note that $z$ satisfies the following differential equation,

$$
\dot{z}=C A x+C B(u+d),
$$

Call $\hat{z}$ an estimate of $z$, and denote the estimation error as

$$
e=z-\hat{z} \text {. }
$$

Construct the governing equation of $\hat{z}$ as follows.

$$
\dot{\hat{z}}=C A x+\beta e+C B(u+v), \quad v=\rho \frac{e}{|e|+\epsilon},
$$

where $\beta$ is a positive constant, $\rho$ an estimator gain larger than the disturbance upper bound $D_{0}$, and $\epsilon$ is a positive constant close to zero. With the above estimator (20), an estimate of the disturbance $d$ will be provided by

$$
\hat{d}=\frac{\beta}{C B} e+v=\frac{\beta e}{C B}+\frac{\rho e}{|e|+\epsilon} .
$$

Once one has obtained an estimate of $d$, one can approximate $s_{2}$ in (18) by

$$
\hat{s}_{2}=C A x+C B(u+\hat{d})+\lambda C x .
$$

The following theorem proves the effectiveness of the above disturbance estimator (20) and (21).

Theorem 2: The disturbance estimation error $d-\hat{d}$, where $\hat{d}$ is given by (21), will become arbitrarily small if the estimator gain $\rho$ in (20) is sufficiently large.

Proof: One can refer to the original paper on disturbance estimator (Chen \& Tomizuka, 1989). For completeness of this chapter, a simple proof will be given below. From (19) - (20), one can easily obtain

$$
\begin{aligned}
\dot{e} & =-\beta e-C B(v-d) \\
& =-\beta e-C B\left(\rho \frac{e}{|e|+\epsilon}-d\right) .
\end{aligned}
$$

It will be shown that both $e$ and $\dot{e}$ will become arbitrarily small if $\rho$ is sufficiently large. Notice from (23) and (21) that $\dot{e}=C B(d-\hat{d})$. Therefore, the smallness of $\dot{e}$ implies the smallness of $d-\hat{d}$ and hence, the success of disturbance estimation. 
Let Lyapunov function $V_{1}=\frac{1}{2} e^{2}$, and take its time derivative,

$$
\begin{aligned}
\dot{V}_{1} & =e \dot{e}=e(-\beta e-C B(v-d)) \\
& \leq-\beta e^{2}-|C B e|\left(\rho \frac{|e|}{|e|+\epsilon}-D_{0}\right) \\
& \leq-\beta n_{1}{ }^{2}, \quad \text { for all } e \notin N_{1},
\end{aligned}
$$

where $N_{1}=\left\{e:|e|<n_{1}=\left(\epsilon D_{0}\right) /\left(\rho-D_{0}\right)\right\}$. With the last inequality, one can prove (see (Chen \& Tomizuka, 1989)) that $|e(t)|<n_{1}$ for all $t>T_{1}$ for some finite time $T_{1}$. Since $n_{1}=\left(\epsilon D_{0}\right) /\left(\rho-D_{0}\right)$ becomes arbitrarily small as the disturbance estimator gain $\rho$ becomes sufficiently large, one concludes that $e$ becomes arbitrarily small within a finite time if $\rho$ is sufficiently large.

To check the behavior of $\dot{e}$, one chooses $V_{2}=\frac{1}{2} \dot{e}^{2}$, and take its time derivative,

$$
\begin{aligned}
\dot{V}_{2} & =\dot{e} \ddot{e}=\dot{e}\left(-\beta \dot{e}-C B\left(\frac{\rho \epsilon \dot{e}}{(|e|+\epsilon)^{2}}-\dot{d}\right)\right) \\
& \leq-\beta \dot{e}^{2}-\frac{\rho \epsilon|C B||\dot{e}|^{2}}{\left(n_{1}+\epsilon\right)^{2}}+|C B \dot{e}| D_{1}, \quad t \geq T_{1}, \\
& \leq-\beta \dot{e}^{2}-\frac{\rho \epsilon|C B \dot{e}|}{\left(n_{1}+\epsilon\right)^{2}}\left(|\dot{e}|-\frac{D_{1}\left(n_{1}+\epsilon\right)^{2}}{\rho \epsilon}\right), t \geq T_{1}, \\
& \leq-\beta n_{2}{ }^{2}, \quad \text { for all } \dot{e} \notin N_{2},
\end{aligned}
$$

where $N_{2}=\left\{\dot{e}:|\dot{e}|<n_{2}=D_{1}\left(n_{1}+\epsilon\right)^{2} /(\rho \epsilon)\right\}$. From the last inequality, one can prove (Chen \& Tomizuka, 1989) that $|\dot{e}(t)|<n_{2}$ for all $t>T_{2}$ for some finite time $T_{2}$. Since $n_{2}=D_{1}\left(n_{1}+\right.$ $\epsilon)^{2} /(\rho \epsilon)$ becomes arbitrarily small as the disturbance estimator gain $\rho$ becomes sufficiently large, one concludes that $\dot{e}=C B(d-\hat{d})$ becomes arbitrarily small within a finite time if $\rho$ is sufficiently large. End of proof.

\subsection{Control variable design}

In the filtered sliding mode control, the objective of the control variable $w=\dot{u}$ is to drive the sliding variable $s_{2}$ to (almost) zero in the face of unknown disturbance. For this purpose, one chooses

$$
\begin{aligned}
\dot{u}= & w \\
= & -\left(C A^{2}+\lambda C A\right) x-(C A B+\lambda C B) u \\
& -\sigma s_{2}-\delta \operatorname{sgn}\left(s_{2}\right),
\end{aligned}
$$

where $\sigma>0, \operatorname{sgn}(\cdot)$ is the sign function, and $\delta$ is an upper bound of the uncertainty $|\Delta p|$ with

$$
\Delta p=(C A B+\lambda C B) d+\dot{d} .
$$

As explained in the previous section, it is impossible to evaluate the sliding variable $s_{2}$ due to the disturbance $d$ involved. Hence, to implement the proposed control, one uses the estimate $\hat{s}_{2}$ in place of $s_{2}$,

$$
\begin{aligned}
\dot{u}= & w \\
= & -\left(C A^{2}+\lambda C A\right) x-(C A B+\lambda C B) u \\
& -\sigma \hat{s}_{2}-\delta \operatorname{sgn}\left(\hat{s}_{2}\right),
\end{aligned}
$$


where $\hat{s}_{2}$ comes from (22). Finally, it is commented that in the above filtered sliding mode control law one can replace the sign function by other smooth approximations such as the saturation function or other boundary layer design.

From (25), the true control input to the system is given by

$$
u=H(s) w, \quad H(s)=\frac{1}{s} .
$$

Even though the switching control $w$ in (25) contains high-frequency chattering, the high-frequency chattering will be filtered out by the low-pass filter $H(s)$. The control input $u$ to the real system can be obtained by direct integration and then becomes chattering free.

The following theorem, which is the main result of this chapter, proves that the proposed control (25) is practically stabilizing.

Theorem 3: The proposed filtered sliding mode control (25) practically stabilizes the system (16) with bounded control $u$, in the sense that the system state is asymptotically driven into a residual set around the origin, with the size of residual set becoming arbitrarily small when the estimator gain $\rho$ in the disturbance estimator (20) becomes sufficiently large.

Proof: Denote $\tilde{s}_{2}=s_{2}-\hat{s}_{2}$, where $s_{2}$ and $\hat{s}_{2}$ are as given by (18) and (22) respectively. It is easy to check that $\tilde{s}_{2}=C B(d-\hat{d})$. To study the evolution of $s_{2}$, choose Lyapunov function $V=\frac{1}{2} s_{2}^{2}$ and check its time derivative under the proposed control $w$ in (25),

$$
\begin{aligned}
\dot{V}= & s_{2}\left[\left(C A^{2}+\lambda C A\right) x+(C A B+\lambda C B) u+C B w\right. \\
& +(C A B+\lambda C B) d+C B \dot{d}] \\
= & s_{2}\left[-\sigma \hat{s}_{2}-\delta \operatorname{sgn}\left(\hat{s}_{2}\right)+\Delta p\right] \\
= & -\sigma s_{2}^{2}+\sigma s_{2} \tilde{s}_{2}+s_{2}\left[-\delta \operatorname{sgn}\left(\hat{s}_{2}\right)+\Delta p\right],
\end{aligned}
$$

where $\Delta p$ is as given in (24), and one has used $\hat{s}_{2}=s_{2}-\tilde{s}_{2}$ to obtain the third equality. There are two possible cases for the square brackets in the above equation.

Case 1. $\left|s_{2}\right|>\left|\tilde{s}_{2}\right|:$ In this case, $\operatorname{sgn}\left(\hat{s}_{2}\right)=\operatorname{sgn}\left(s_{2}-\tilde{s}_{2}\right)=\operatorname{sgn}\left(s_{2}\right)$. Equation (27) then becomes

$$
\begin{aligned}
\dot{V} & \leq-\sigma s_{2}^{2}+\sigma s_{2} \tilde{s}_{2}-\left|s_{2}\right|(\delta-|\Delta p|) \\
& \leq-\sigma s_{2}^{2}+\sigma s_{2} \tilde{s}_{2} \\
& \leq-\sigma\left|s_{2}\right|^{2}+\sigma\left|s_{2}\right| v,
\end{aligned}
$$

where the second inequality results from the design choice $\delta>|\Delta p|$, and the third inequality (with $v$ an arbitrarily small number) comes from Theorem 2 that $\tilde{s}_{2}=C B(d-\hat{d})$ becomes arbitrarily small asymptotically. From the last inequality, it is not difficult to show that asymptotically one has $\lim _{t \rightarrow \infty}\left|s_{2}\right| \leq v$; that is, $s_{2}$ becomes arbitrarily small asymptotically.

Case 2. $\left|s_{2}\right| \leq\left|\tilde{s}_{2}\right|$ : Since $\tilde{s}_{2}=C B(d-\hat{d})$, it follows from Theorem 2 that $\left|s_{2}\right|$ becomes arbitrarily small asymptotically.

Judging from conclusions of both Case 1 and 2, one can say that the sliding variable $s_{2}$ becomes arbitrarily small asymptotically. One next shows that the system state $x$ will also become arbitrarily small as $s_{2}$ does. To this end, introduce a state transformation (Isidori, 1989),

$$
x=T\left[\begin{array}{l}
z \\
\eta
\end{array}\right], \quad T \in R^{n \times n} .
$$

where the external state $z \in R^{1}$ is as defined in (17), and the internal state $\eta \in R^{n-1}$ satisfies

$$
\dot{\eta}=Q \eta+L z
$$


for some matrices $Q, L$, in which $Q$ is a square matrix whose eigenvalues are open-loop zeros of the triple $(A, B, C)$ (Isidori, 1989). Since, in the design of sliding variable in (17), $z=C x$ is chosen such that $(A, B, C)$ has only stable zeros, $Q$ is stable.

When $s_{2}$ becomes arbitrarily small, it follows from (17) that the external state $z$ also becomes arbitrarily small since $\dot{z}+\lambda z=s_{2}$ can be regarded as a stable system $z$ subject to small input signal $s_{2}$. Similarly, (29) can be regarded as a stable system $\eta$ subject to small input signal $z$. Hence, its state $\eta$ will also become arbitrarily small asymptotically. Finally, since both $z$ and $\eta$ become arbitrarily small, so does the original system state $x$ according to the state transformation (28). End of proof.

To show the efficacy of the proposed filtered sliding mode control in noisy environments, a simulation example is presented below.

\section{Example 2: Filtered sliding mode control.}

The same system as in Example 1 is tested again for the proposed filtered sliding mode control (25). Here one has chosen

$$
C=[2,3,1] \text {. }
$$

The disturbance $d=\cos (t)$ and the state measurement is contaminated with a uniform noise with zero-mean and standard deviation 0.05 . One tests the proposed filtered sliding mode control (25). The parameters are chosen such that $\lambda=2$ in (17) and $\beta=100, \rho=1.2, \epsilon=0.005$ in (20). Other design parameters are $\sigma=30$ and $\delta=9.6$ in (25). The plot of Figure 4(a) shows the time history of system state, which achieves almost the same performance as that with the boundary layer control. However, note from the plot of Figure 4(b) that the filtered sliding mode design has successfully removed chattering in the control input $u$ even in this noisy environment.

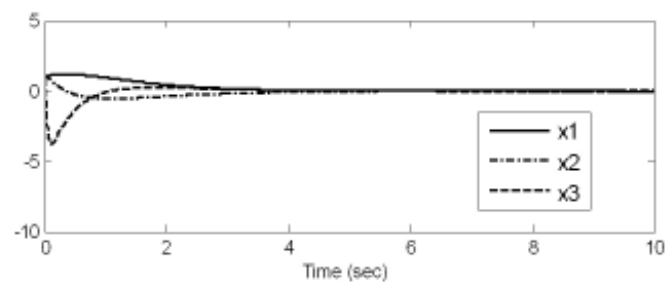

(a) System state

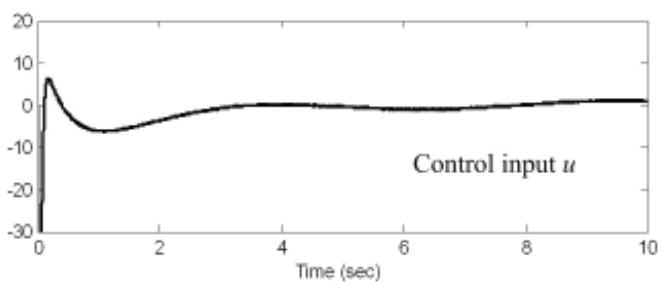

(b) Control input

Fig. 4. Filtered sliding mode control with noise 


\section{Conclusions}

This chapter first shows via the linearization technique and the frequency domain analysis that the boundary layer design in the sliding mode control can still exhibit control chattering due to the excitations of measurement noise. Hence, other solutions to the chattering reduction such as those in (Chen et al., 2002; 2007) should be searched.

Second, a new design is proposed to reduce control chattering in sliding mode control by low-pass filtering the control signal. The new design requires estimation of the sliding variable, and this is achieved by the use of a disturbance estimator. The unique feature of this new design is that chattering reduction is achieved by low-pass filtering the control signal, and control accuracy can be maintained by a sufficiently large disturbance estimator gain. This is contrary to the conventional boundary layer design, where chattering reduction is achieved at the price of sacrificing the control accuracy. This chapter further shows via simulation examples that when there is high-level measurement noise, the boundary layer design can no longer reduce chattering, but the new design in this chapter can effectively reduce chattering even in noisy environments.

\section{References}

Bartolini, G. (1989). Chattering phenomena in discontinuous control systems, Int. J. Systems Sci., Vol. 20, 2471-2481.

Bartolini, G., \& Pydynowski, P. (1996). An improved, chattering free, V.S.C scheme for uncertain dynamical systems, IEEE Trans. Autom. Control, 41, 1220-1226.

Burton, J. A. \& Zinober, A. S. I. (1986). Continuous approximation of variable structure control. Int. J. System Science, 17, 875-885.

Chen, M. S. \& Tomizuka, M. (1989). Disturbance estimator and its application in estimation of system output derivatives, Proceedings of Conference on Decision and Control, Tampa, pp. 452-457.

Chen, M. S., Hwang, Y. R. \& Tomizuka, M. (2002). A state-dependent boundary layer design for sliding mode control. IEEE Trans. Autom. Control, 47, 1677-1681.

Chen, M. S., Chen, C. H. \& Yang, F. Y. (2007). An LTR-observer-based dynamic sliding mode control for chattering reduction. Automatica, 43, 1111-1116.

Corless, M. J., \& Leitmann, G. (1981). Continuous state feedback guaranteeing uniform ultimate boundedness for uncertain dynamic systems, IEEE Trans. Autom. Control, 26, 1139-1143.

Hung, J. Y., Gao, W. B. \& Hung, J. C. (1993). Variable structure control: a survey. IEEE Trans. Ind. Electron., 40, 2-22.

Isidori, A. (1989) Nonlinear Control Systems, Springer-Verlag, New York.

Sira-Ramirez, H. (1993). On the dynamical sliding mode control of nonlinear systems, Int. J. Control, 57, 1039-1061.

Sira-Ramirez, H., Llanes-Santiago, O. \& Fernadez, N. A. (1996). On the stabilization of nonlinear systems via input-dependent sliding surfaces, Int. J. of Robust and Nonlinear Control, 6, 771-780.

Slotine, J. J. E. \& Sastry, S. S. (1983). Tracking control of nonlinear systems using sliding surfaces with application to robot manipulator. Int. J. Contr., 38, 931-938.

Utkin, V. I. (1977). Variable structure systems with sliding modes. IEEE Trans. Autom. Control, AC-22, 212-222. 
Xu, J.-X., Pan, Y.J. \& Lee, T.H. (2004). A new sliding mode control with closed-loop filtering architecture for a class of nonlinear systems, IEEE Transactions on Circuits and Systems II: Express Briefs, 51 (4), 168-173. 


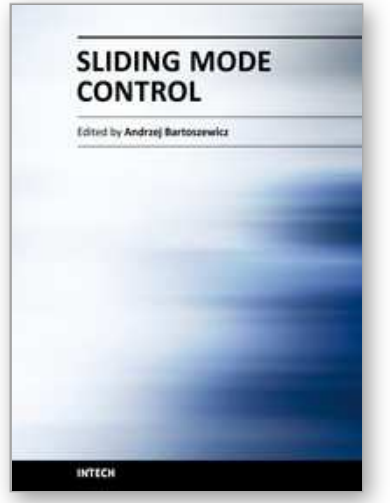

\author{
Sliding Mode Control \\ Edited by Prof. Andrzej Bartoszewicz
}

ISBN 978-953-307-162-6

Hard cover, 544 pages

Publisher InTech

Published online 11, April, 2011

Published in print edition April, 2011

The main objective of this monograph is to present a broad range of well worked out, recent application studies as well as theoretical contributions in the field of sliding mode control system analysis and design. The contributions presented here include new theoretical developments as well as successful applications of variable structure controllers primarily in the field of power electronics, electric drives and motion steering systems. They enrich the current state of the art, and motivate and encourage new ideas and solutions in the sliding mode control area.

\title{
How to reference
}

In order to correctly reference this scholarly work, feel free to copy and paste the following:

Min-Shin Chen and Ming-Lei Tseng (2011). A New Design for Noise-Induced Chattering Reduction in Sliding Mode Control, Sliding Mode Control, Prof. Andrzej Bartoszewicz (Ed.), ISBN: 978-953-307-162-6, InTech, Available from: http://www.intechopen.com/books/sliding-mode-control/a-new-design-for-noise-inducedchattering-reduction-in-sliding-mode-control

\section{INTECH}

open science | open minds

\section{InTech Europe}

University Campus STeP Ri

Slavka Krautzeka 83/A

51000 Rijeka, Croatia

Phone: +385 (51) 770447

Fax: +385 (51) 686166

www.intechopen.com

\section{InTech China}

Unit 405, Office Block, Hotel Equatorial Shanghai

No.65, Yan An Road (West), Shanghai, 200040, China

中国上海市延安西路65号上海国际贵都大饭店办公楼 405 单元

Phone: +86-21-62489820

Fax: +86-21-62489821 
(C) 2011 The Author(s). Licensee IntechOpen. This chapter is distributed under the terms of the Creative Commons Attribution-NonCommercialShareAlike-3.0 License, which permits use, distribution and reproduction for non-commercial purposes, provided the original is properly cited and derivative works building on this content are distributed under the same license. 